\title{
Computed tomographic colonography without cathartic preparation performed well in detecting colorectal polyps
}

lannaccone R, Laghi A, Catalano C, et al. Computed tomographic colonography without cathartic preparation for the detection of colorectal polyps. Gastroenterology 2004;127:1300-11.

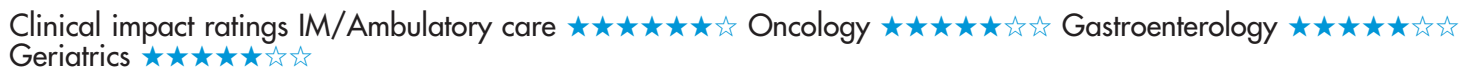

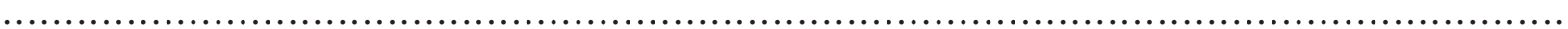

What is the diagnostic performance of low dose multidetector computed tomographic colonography (CTC) without bowel cathartic preparation compared with optical colonoscopy for detection of colonic polyps?

\section{METHODS}

ए

Design: blinded comparison of CTC with optical colonoscopy

(19)

Setting: a university hospital in Rome, Italy.

Patients: 203 patients $\geqslant 35$ years of age (mean age 61 y, 69\% men) who were scheduled to have optical colonoscopy because

of average risk colorectal cancer screening, personal or family history of colorectal polyps, family history of colorectal cancer, abnormal screening test result, iron deficiency anaemia, haematochezia, change in bowel habits, abdominal pain, or weight loss. Exclusion criteria were history of familial adenomatous polyposis or hereditary non-polyposis cancer syndromes; previous colorectal surgery; suspected inflammatory bowel disease, bowel obstruction, or acute diverticulitis; contraindications to bowel preparation or iodine containing contrast agents; or pregnancy

Description of test: foecal tagging was done using an ora iodinated contrast agent, which patients ingested during the 5 principal meals 48 hours before CTC. CTC was done using a multidetector helical CT scanner. The colon was insufflated with room air with patients in the prone and supine positions. CT images were acquired using a low dose protocol for the scanner (slice collimation $2.5 \mathrm{~mm}$, slice thickness $3.0 \mathrm{~mm}$, and reconstruction interval $1.0 \mathrm{~mm}$ ). The images were read separately and independently by 3 gastrointestinal radiologists who were blinded to the indications and results of optical colonoscopy.

Diagnostic standard: optical colonoscopy was done 3-7 days after CTC. A single colonoscopist, blinded to CTC results, inserted a standard video colonoscope into the caecum and sequentially withdrew it segment by segment. After each segment was examined, the results of CTC were revealed for the previously examined segment allowing the endoscopist to reexamine that segment.

Outcomes: sensitivity and specificity of CTC averaged across the 3 readers for detection of colorectal polyps.

For correspondence: Dr R lannaccone, University of Rome, Rome, Italy r_iannaccone@yahoo.it

Source of funding: no external funding.

Test characteristics of computed tomographic colonography (CTC) for detecting colorectal polyps averaged across 3 readers*

\begin{tabular}{lllll}
\hline Test & $\begin{array}{l}\text { Sensitivity } \\
(95 \% \mathrm{CI})\end{array}$ & Specificity (CI) & + LR & $-\mathbf{L R}$ \\
\hline CTC & $90 \%(86$ to 94$)$ & $92 \%(90$ to 95$)$ & 11.5 & 0.11 \\
\hline *Diagnostic terms defined in glossary; LRs calculated from data in article.
\end{tabular}

\section{MAIN RESULTS}

Agreement among the 3 readers for detection of colorectal polyps was high to excellent ( $\kappa$ range 0.79 to 0.91 ). The table shows the diagnostic performance for CTC averaged across the 3 readers. In a per polyp analysis, the mean sensitivity for detecting polyps $(\leqslant 5$ to $\geqslant 10 \mathrm{~mm}$ ) was $64 \%$ (95\% CI 60 to 69 ). The mean sensitivity for polyps $\geqslant 6 \mathrm{~mm}$ was $86 \%$ (CI 82 to 91 ) and for polyps $\geqslant 8 \mathrm{~mm}$ was $96 \%$ (CI 92 to 99$)$.

\section{CONCLUSION}

Low dose multidetector computed tomographic colonography without bowel cathartic preparation compared favourably with optical colonoscopy for detection of colonic polyps.

Abstract and commentary also appear in ACP Journal Club.

\section{Commentary}

$\mathrm{V}$ irtual colonoscopy, considered promising technology for colon cancer screening for over 10 years, has recently begun to live up to its promise. In virtual colonoscopy, an abdominal CT scan is rendered into a 3 dimensional image that looks like the image (minus mucosal coloration and other detail) from conventional colonoscopy. High sensitivity (94\%) and specificity (96\%) have recently been shown, although it is unclear whether such results can be routinely obtained in other settings. ${ }^{2} 3$ A laxative preparation, understandably unpopular with patients, has been needed for conventional colonoscopy and, until now, for virtual colonoscopy. The study by lannaccone et al shows that satisfactory images may be obtained using a non-cathartic tagging technique and is an important advance. ${ }^{4}$

Before virtual colonoscopy becomes well accepted as a colorectal cancer screening test, other challenges will need to be addressed. Because $>30 \%$ of Americans $>50$ years of age have $\geqslant 1$ polyp, decisions must be made about what size polyp is a target for screening, requiring further workup with conventional colonoscopy. Can some small polyps be ignored or simply followed with watchful waiting? ${ }^{3}$ Radiation dose, expense, and inconvenience may be important factors inhibiting the adoption of virtual colonoscopy screening. Currently recommended screening programmes include foecal occult blood testing and sigmoidoscopy as well as colonoscopy. Based on the progress reported recently, however, virtual colonoscopy is likely to become a contender in the near future.

David F Ransohoff, MD University of North Carolina at Chapel Hill Chapel Hill, North Carolina, USA

1 Pickhardt PJ, Choi JR, Hwang I, et al. Computed tomographic virtual colonoscopy to screen for colorectal neoplasia in asymptomatic adults. N Engl J Med 2003;349:2191-200.

2 Cotton PB, Durkalski VL, Pineau BC, et al. Computed tomographic colonography (virtual colonoscopy): a multicenter comparison with standard colonoscopy for detection of colorectal neoplasia. JAMA 2004;291:1713-9.

3 Ransohoff DF. Virtual colonoscopy-what it can do vs what it will do. JAMA 2004;291:1772-4.

4 McFarland EG, Zalis ME. CT colonography: progress toward colorectal evaluation without catharsis. Gastroenterology 2004;127:1623-6. 